\title{
Un changement de culture politique
}

L'éducation à la citoyenneté en Angleterre

"A change in the political culture ". Citizenship education in England

Un cambio de cultura política. Educación a la ciudadanía en Inglaterra

\section{David Kerr}

Traducteur : Yves Goujon, Maroussia Raveaud et Marie-José Sanselme

\section{(2) OpenEdition}

\section{Journals}

Édition électronique

URL : https://journals.openedition.org/ries/128

DOI : $10.4000 /$ ries. 128

ISSN : 2261-4265

Éditeur

France Education international

Édition imprimée

Date de publication : 1 avril 2007

Pagination : $35-44$

ISBN : 978-2-85420-569-5

ISSN : 1254-4590

\section{Référence électronique}

David Kerr, «Un changement de culture politique », Revue internationale d'éducation de Sèvres [En ligne], 44 | avril 2007, mis en ligne le 23 juin 2011, consulté le 06 juillet 2021. URL : http:// journals.openedition.org/ries/128; DOI : https://doi.org/10.4000/ries.128 


\section{Un changement de culture politique}

\section{L'éducation à la citoyenneté en Angleterre*}

\section{David Kerr}

Ces deux dernières décennies ont été marquées par une réflexion fondamentale sur la notion de citoyenneté et sur sa signification dans la société moderne du Royaume-Uni, en Europe et à l'échelle mondiale. Cette recherche a déclenché des débats sur le moyen de préparer les jeunes à être des membres à part entière de la société et sur le rôle de l'éducation dans ce processus.

La réflexion fondamentale sur la notion de citoyenneté a été provoquée par la rapidité du changement dans les sociétés modernes et le besoin d'y réagir. L'Angleterre a entamé un débat particulier à la suite des attentats commis à Londres, en juillet 2005, par des citoyens britanniques de naissance. Les questions portaient sur la façon dont l'éducation pouvait s'orienter vers l'identité et la diversité et sur ce qu'il fallait faire pour renforcer l'esprit de solidarité dans la société britannique $\left(D f E S^{1}, 2007\right)$. Les tentatives pour donner une nouvelle définition à la notion de citoyenneté ont supposé une réforme analogue de l'éducation à la citoyenneté, puisque les deux vont de pair. Ce n'est pas un hasard si l'objectif prioritaire des systèmes éducatifs est une éducation efficace et vivante à la citoyenneté, soumise à l'évaluation des programmes en cours dans de nombreux pays.

\section{L'INTRODUCTION DE L'ÉDUCATION À LA CITOYENNETÉ DANS LES PROGRAMMES}

L'éducation à la citoyenneté a pris de plus en plus d'importance dans les programmes politiques ces dix dernières années en Angleterre et est devenue objet de recherches et d'initiatives. La recherche a été menée sous la tutelle de la Commission consultative sur l'éducation à la citoyenneté et l'enseignement des principes démocratiques à l'école ${ }^{2}$, commission mise en place en 1997 sous la direction du professeur Bernard Crick (Crick, 1998), anobli depuis. Le groupe Crick (comme on le nomme le plus souvent) a fixé les objectifs et les missions de l'éducation à la citoyenneté et l'enseignement de la démocratie à l'école; il a proposé un schéma directeur pour la mise en œuvre, tant au sein qu'à l'extérieur des programmes scolaires officiels, à travers les liens entre l'école et la société.

\footnotetext{
* Article traduit par Yves Goujon, Maroussia Raveaud et Marie-José Sanselme.

1. Department for Education and Skills, équivalent en France du ministère de l'éducation.

2. Advisory Group on Education for Citizenship and the Teaching of Democracy in Schools.
} 


\section{Une nouvelle matière obligatoire}

La Commission consultative sur la citoyenneté ${ }^{3}$ s'est proposé de renforcer l'éducation à la citoyenneté et a ainsi défini une «éducation efficace à la citoyenneté » comprenant trois axes distincts mais liés :

- la responsabilité sociale et morale avec «... l'apprentissage précoce par l'enfant de la confiance en soi et d'un comportement responsable tant au plan social que moral, à l'école et au-delà de la salle de classe, vis-à-vis de ceux qui détiennent l'autorité et de leurs camarades »; cet axe préliminaire aux deux autres joue un rôle essentiel;

- l'implication dans le groupe par «... l'apprentissage et l'acquisition de comportements utiles pour la vie et les problèmes de leurs communautés, ce qui inclut un apprentissage par implication dans la communauté et un service à la communauté»; ceci, tout comme les deux autres axes, ne se limite en aucun cas au temps passé à l'école;

- une culture politique : «les élèves apprennent pourquoi et comment devenir des membres efficaces de la vie publique grâce aux connaissances, compétences et valeurs enseignées. » L'expression «vie publique» est utilisée ici dans son acception la plus large et englobe une connaissance réaliste et une préparation à la résolution de conflits et à la prise de décision, face à des problèmes au niveau local, national, européen, ou mondial.

Le rapport final de la commission Crick contient l'audacieuse déclaration suivante : l'objectif central du renforcement de l'éducation à la citoyenneté est d'opérer «rien moins qu'un changement de culture politique de ce pays, tant au plan national que local» (Crick, 1998 p. 7).

Le rapport de la commission fut bien reçu et l'éducation à la citoyenneté fut insérée pour la première fois dans les programmes scolaires des enfants âgés de cinq à seize $\left(Q C A^{4}, 1999\right)$. La citoyenneté fait partie maintenant des recommandations (sans caractère obligatoire) pour l'éducation personnelle, sociale et sanitaire $\left(\mathrm{PSHE}^{5}\right.$ ) et la citoyenneté dans l'enseignement primaire (pour des enfants âgés de cinq à onze ans), et elle constitue une matière obligatoire dans l'enseignement secondaire. On a par conséquent exigé légalement des écoles qu'elles délivrent une éducation à la citoyenneté à partir de septembre 2002 .

Les programmes pour l'enseignement secondaire précisent l'importance de la citoyenneté en tant que nouvelle matière reconnue en Angleterre. Elle apporte "aux élèves, les connaissances, les compétences et l'entendement leur permettant de jouer un rôle efficace dans la société aux niveaux local,

\footnotetext{
3. The Citizenship Advisory Group.

4. Qualification Curriculum Authority.

5. Personal, Social And Health Education.
} 
national, et international. Elle les aide à devenir des citoyens informés, réfléchis et responsables qui sont conscients de leurs devoirs et de leurs droits... Elle encourage les élèves à se comporter en membres utiles à la vie de leurs écoles, de leurs quartiers, des communautés et plus largement du monde. Elle leur enseigne également notre système économique, nos institutions démocratiques et nos valeurs. Elle encourage le respect des identités ethniques, religieuses et nationales différentes. Elle développe la capacité des élèves à réfléchir sur des problèmes et à prendre part à des discussions.» (QCA, 1999 p. 12).

Les nouvelles instructions offrent des programmes d'études de la citoyenneté et des objectifs fondés sur trois éléments :

- la connaissance et la compréhension de la question "pourquoi devenir des citoyens avisés?»;

- le développement de compétences en matière de recherche et de communication ;

- le développement des capacités de participation et d'action responsable.

Ces trois éléments sont intentionnellement liés entre eux afin que l'enseignement garantisse que «la connaissance et la compréhension de la question “pourquoi devenir des citoyens avisés" " soient acquises et appliquées au moment du «développement des techniques d'enquête et d'approche et des capacités de participation et d'action responsable.»

Les programmes diffèrent de ceux des autres matières car ils sont délibérément «esquissés». Ils proposent un cadre général mais rigoureux de ce qui doit être enseigné et appris, mais laissent ensuite s'appliquer le jugement professionnel de ceux qui - directeurs, coordinateurs et enseignants -, travaillant en partenariat avec les collectivités locales, décident de la meilleure manière de l'aborder. Il n'y a pas de directive officielle sur la façon dont les lycées et les écoles doivent aborder la citoyenneté.

\section{Des élargissements nécessaires}

Le processus politique a considérablement évolué depuis 2002, tant en termes d'éducation à la citoyenneté qu'en termes d'éducation, au sens général. Il a été marqué par deux évolutions. Tout d'abord, les politiques gouvernementales ont facilité considérablement, et de plus en plus, l'implication des individus citoyens, y compris les enfants et les jeunes gens, dans les principales institutions (telles que les écoles) et les communautés qui jouent un rôle dans leur vie. Ensuite, l'accent a été mis, suite aux attentats de Londres, sur le rôle de l'éducation, et de l'éducation à la citoyenneté en particulier, pour aider les jeunes gens à mieux comprendre les questions de diversité et d'identité, posant ainsi les fondements d'une plus grande cohésion sociale. Ceci a entraîné des directives récentes en faveur de plus de débats dans les écoles sur ce que 
signifie «vivre ensemble» dans une Grande-Bretagne moderne, au regard de la race, de l'histoire, de l'identité, de la culture, des valeurs et des appartenances diverses.

L'accent mis sur la capacité d'influence des citoyens via les institutions et les communautés est le pendant d'une politique d'éducation à la citoyenneté qui dépasse, depuis 2002, le seul cadre scolaire et comprend d'autres temps d'éducation et de formation dans des cercles plus larges.

Pendant ce temps, le Home Office ${ }^{6}$ a lancé une initiative politique majeure autour d'un renouveau civique (Blunkett, 2003) qui, selon lui, doit comprendre trois éléments essentiels :

- la «citoyenneté active» de ceux qui s'engagent pour s'attaquer aux problèmes qu'ils observent dans leur propre communauté;

- le «renforcement des communautés» qui peuvent créer et maintenir leurs propres organisations, œuvrant ainsi à rapprocher les individus et à leur permettre de gérer ensemble leurs problèmes communs;

- des "partenariats sur les besoins publics», avec la collaboration de citoyens et d'organismes publics en matière d'amélioration du service public.

Par ailleurs, l'évolution de la société du Royaume-Uni a mis en relief les questions d'immigration, de diversité, de différence, d'identité, de «valeurs communes", et de cohésion sociale. Le fait que les auteurs des attentats de Londres de 2005 soient des musulmans britanniques de naissance a catalysé l'attention sur la manière dont il faudrait que ces questions soient abordées. En conséquence, depuis novembre 2006, les écoles ont reçu pour mission de promouvoir la cohésion sociale. La dernière version du chapitre sur la diversité et la citoyenneté dans les programmes scolaires (DfES, 2007; Maylor et Read et al., 2007) propose un certain nombre de recommandations pour renforcer l'approche de la diversité, de la race et de l'identité ethnique. Elles comprennent notamment l'introduction d'un quatrième axe explicite dans le programme d'éducation à la citoyenneté, intitulé «identité et diversité : vivre ensemble au Royaume-Uni» et du temps consacré à la discussion sur la question centrale : «qui pensons-nous être?»

\section{De nOUVEAUX CONSTATS}

Comme nous l'avons dit, l'introduction en 2002 de l'éducation à la citoyenneté dans les programmes nationaux comme nouvelle matière obligatoire a marqué le début plutôt que la fin d'un processus politique en Angleterre. En effet, l'éducation à la citoyenneté continue de susciter l'intérêt des décideurs politiques, des praticiens et des journalistes cinq ans après son introduction dans les écoles. Quelles leçons pouvons-nous aujourd'hui en tirer?

6. Ministère de l'Intérieur. 
L'apparition de nouvelles définitions et de nouvelles approches officielles dans les programmes a été la principale préoccupation, en particulier du fait de l'absence d'une tradition d'éducation à la citoyenneté dans les programmes en Angleterre. Des questions revenaient sans cesse telles que : «à quel point l'éducation à la citoyenneté a-t-elle été bien comprise par les administrateurs et les professeurs? Comment est-elle enseignée dans les écoles et les lycées, et avec quelles compétences? Quels sont les questions et défis qui en résultent pour les écoles, les lycées et les professeurs? Comment peuvent-ils être surmontés?» (Kerr et al., 2004; Kerr \& Cleaver, 2004; Deakin-Crick et al., 2004).

\section{Des politiques aux pratiques}

Les décideurs politiques reconnaissent que le rapport du groupe Crick et le nouveau programme Instructions pour une citoyenneté ${ }^{7}$ sont en euxmêmes insuffisants pour encourager le développement d'une éducation à la citoyenneté efficace à l'école et au-delà. Une stratégie de mise en œuvre systématique et concertée est nécessaire afin de combler le fossé entre les décisions politiques et les pratiques et de poser les fondations du développement de pratiques efficaces.

L'Angleterre en est encore aux balbutiements de l'éducation à la citoyenneté et les décideurs politiques et les organismes impliqués travaillent sans relâche pour répondre aux besoins grandissants des directeurs d'établissements scolaires, des coordinateurs et des professeurs en matière d'introduction de cette nouvelle matière, la citoyenneté. En conséquence, ces cinq dernières années, des actions ont été engagées dans quatre secteurs stratégiques, à savoir :

- la rédaction de guides et de conseils plus détaillés pour les écoles, les lycées et les professeurs;

- le financement de la production de ressources pour combler les lacunes identifiées par les professeurs dans le programme national obligatoire de cette nouvelle matière;

- l'aide au développement d'échanges de bonnes pratiques professionnelles;

- un soutien fort à la recherche et aux bases de données sur l'éducation à la citoyenneté ;

- avec une éducation à la citoyenneté passant rapidement du statut de proposition politique à celui de véritable matière enseignée est né le besoin de renforcer cette base de recherche. En conséquence, le DfES a demandé à la fondation nationale pour la recherche éducative (NFER) d'entreprendre une

7. Order for Citizenship. 
étude de huit ans sur l'éducation à la citoyenneté, afin d'en mesurer les effets à court et à long terme sur les savoirs, la compréhension, les attitudes et les comportements des élèves.

\section{La recherche et les résultats déjà rassemblés}

La recherche et les résultats déjà rassemblés permettent d'apporter des réponses aux questions sur la définition de l'éducation à la citoyenneté sur laquelle travaillent les écoles et les collèges, sur leur façon d'aborder cette nouvelle matière et les défis qu'ils relèvent dans leur effort pour transformer une volonté politique en réelle pratique de classe. Ils permettent de jeter un éclairage réaliste sur l'état actuel de l'éducation à la citoyenneté dans les établissements anglais.

\section{Des programmes aux pratiques}

On observe un fossé entre la vision des politiques, telle qu'elle est présentée par le groupe Crick et dans différents programmes d'éducation à la citoyenneté, et la capacité des acteurs de l'école à la comprendre, à se l'approprier et à la mettre en pratique. Ce fossé s'observe non seulement au niveau national, mais aussi au niveau des établissements scolaires. Les résultats de l'étude de l'éducation à la citoyenneté révèlent des différences d'appréciation entre les directeurs d'établissements scolaires, les professeurs et les élèves vis-à-vis du contenu enseigné (Kerr et al., 2004; Ireland et al., 2006). Les directeurs sont les plus positifs en ce qui concerne les pratiques dans les écoles ou les lycées, c'est-à-dire en ce qui concerne «ce qui est prévu»; les professeurs le sont moins que les directeurs d'école, c'est-à-dire en ce qui concerne «ce qui est réellement enseigné » et les élèves sont les moins positifs sur les pratiques, c'est-à-dire sur «ce qu'ils reçoivent réellement». Le fossé entre le contenu proposé, la façon dont il est enseigné et celle dont il est reçu au niveau national et institutionnel existera probablement toujours; le défi est de le ramener à un niveau acceptable.

\section{D’autres définitions possibles}

On note ensuite une redéfinition de l'éducation à la citoyenneté qui s'éloigne des trois axes du rapport du groupe Crick (responsabilité sociale et morale, implication dans la communauté et culture politique) et tend vers une conceptualisation croissante comprenant trois aspects étroitement liés :

- la citoyenneté dans les programmes : l'enseignement d'une discipline autonome en lien avec d'autres disciplines et par des travaux pratiques et des activités hors programme;

- la citoyenneté active dans la culture scolaire : l'articulation avec des processus démocratiques plus larges et avec l'ensemble de l'institution offrant la 
possibilité aux élèves de participer par le biais de structures officielles comme les conseils d'école, et par des pratiques quotidiennes informelles;

- la citoyenneté active avec la communauté élargie : les liens de l'école avec ses partenaires au niveau local, mais aussi national et international. Ceux qui sont sur le terrain trouvent plus utile de parler de ces trois aspects de la citoyenneté, car ils sont plus adaptés à la réalité des pratiques quotidiennes dans les établissements scolaires.

\section{Une situation disparate}

Même en tenant compte du caractère inégal, disparate et variable des résultats actuels de la recherche, il semble que de nouvelles approches de l'éducation à la citoyenneté soient en train d'émerger à l'école et au lycée.

Cependant, une étude menée auprès d'un échantillon représentatif au niveau national (Kerr et al., 2004) souligne, dans trois-quarts des établissements observés, la grande faiblesse des approches retenues en matière d'éducation à la citoyenneté, notamment si on les compare aux objectifs fixés par les instructions officielles. Une explication serait que, en étant à ses balbutiements, dans les trois quarts des écoles sondées, l'éducation à la citoyenneté a connu un début prometteur et elle dispose d'un potentiel considérable de développement et d'amélioration. C'est le dilemme classique du verre à moitié plein ou à moitié vide, appliqué aujourd'hui aux approches de l'éducation à la citoyenneté à l'école.

\section{Les facteurs à prendre en compte}

Il s'agit d'identifier, sur la base d'un consensus général, les facteurs clés qui sont susceptibles de soutenir, promouvoir et relever le défi de l'éducation à la citoyenneté (Kerr et al., 2004a; Craig et al., 2004). Les établissements qui semblent le mieux y parvenir partagent deux séries de caractéristiques. Au niveau de l'établissement, tout d'abord, ils font preuve d'un engagement fort de la part de l'administration, d'une compréhension fine de ce que recouvre l'éducation à la citoyenneté et d'initiatives alliant les trois éléments interdépendants que sont la citoyenneté dans les programmes, la citoyenneté active à l'école et dans la communauté au sens large. En matière d'apprentissages, ensuite, ils bénéficient de personnels enthousiastes et compétents déployant un éventail d'approches pédagogiques, et impliquent les élèves dans la vie sociale de l'établissement par des structures et initiatives fondées sur la confiance, le respect et le dialogue (par exemple les conseils d'école et de classe, les médiateurs et les activités extrascolaires).

Ces facteurs laissent supposer que le développement de l'éducation à la citoyenneté ne résulte pas d'un facteur particulier mais plutôt de la combinaison complexe d'un ensemble de facteurs, d'influences et d'individus. 


\section{Les défis}

Il reste un certain nombre de questions-clés et de défis à relever afin que les apports de l'éducation à la citoyenneté deviennent plus visibles, plus cohérents et plus efficaces : sont concernés, entre autres, les problèmes de définition, d'appropriation, de formation et de développement, d'évaluation et la question de la citoyenneté active.

Il faut entre autres proposer des formations adéquates aux professeurs. La question de l'évaluation et des rapports d'étape reste préoccupante car elle n'est pas encore vraiment prise en compte dans les établissements et les enseignants manquent encore de confiance en eux sur ce point. Les établissements trouvent également que la citoyenneté active est l'une des notions les plus difficiles à développer et à mettre en œuvre, plus particulièrement en ce qui concerne la communauté élargie. Des interrogations se sont fait jour sur la capacité des établissements et des autres institutions à proposer à tous les jeunes de réelles occasions de pratiquer une «vraie» citoyenneté, étant donné les cultures et les structures dominantes qui demeurent très hiérarchisées et antidémocratiques. Des questions subsistent sur ce que signifie «éducation à la citoyenneté» non seulement pour les directeurs d'écoles et les enseignants, mais aussi les élèves.

Il est intéressant de noter que toutes ces questions - comment passer des programmes aux pratiques, comment comprendre les résultats des recherches en cours et de la base de données - sont déjà examinées attentivement par les décideurs politiques, les organismes associés et ceux qui sont sur le terrain, dans la perspective d'y répondre.

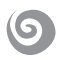

Alors que les évolutions actuelles dans les politiques et pratiques d'éducation à la citoyenneté en Angleterre alimentent considérablement la réflexion dans les autres pays, il est important qu'elles soient perçues dans le cadre plus large de la révision de l'éducation à la citoyenneté qui se joue à l'échelle mondiale. Les évolutions en Angleterre sont une réaction rapide à un défi commun : comment développer chez les jeunes le sentiment qu'ils appartiennent à la société, en tant qu'individus et en collaboration avec d'autres, et qu'ils ont le pouvoir de s'y impliquer. On pourra évaluer l'éducation à la citoyenneté quand on pourra déterminer dans quelle mesure elle réussit à construire cette notion d'efficacité de l'élève chez les jeunes, particulièrement à l'école, comme le montre l'IEA dans son étude sur l'éducation civique (TorneyPurta et al., 2001; Kerr et al., 2002), mais aussi à la maison et dans la société civile et politique. Si nous parvenons à atteindre ce but, les jeunes nous aideront alors à redéfinir l'éducation à la citoyenneté au cours de ce processus. Cependant, ce ne sera pas une tâche aisée. 
Ces remises en cause n'ont pas lieu qu'en Angleterre, mais partout où existe une réflexion, une révision, une mise en œuvre et à nouveau une révision de l'éducation à la citoyenneté. Il faut également bien comprendre que ces remises en cause sont permanentes, toujours en cours et qu'elles ne sont pas exceptionnelles. Le renforcement actuel de l'éducation à la citoyenneté en Angleterre, lié à des questions d'identité, de diversité et de cohésion sociale fournit un exemple concret de la nécessité d'une réactualisation ultérieure pour répondre aux défis actuels.

Seul le temps nous dira si nos réponses sont bien adaptées aux conséquences du changement sociétal en Angleterre. La preuve en sera apportée par les attitudes, les actions et les comportements des jeunes. C'est seulement alors que nous pourrons juger dans quelle mesure l'objectif central du renforcement de l'éducation à la citoyenneté, tel qu'il est fixé par le groupe Crick, pour opérer «rien moins qu'un changement dans la culture politique de ce pays au niveau national et local», a été atteint. Tout ce que nous pouvons dire aujourd'hui, c'est qu'il y a eu un démarrage significatif. Bien que nous ayons parcouru un long chemin depuis dix ans, nous en sommes toujours au début de l'éducation à la citoyenneté en Angleterre. Ce n'est que lorsqu'une génération de jeunes aura suivi un cursus obligatoire complet d'éducation à la citoyenneté à l'école qu'un jugement valable sur son impact général sera possible. Quelle que soit la conclusion finale, il faudra souligner que nous aurons au moins essayé d'apporter des changements positifs à travers l'éducation à la citoyenneté.

\section{BIBLIOGRAPHIE}

BLUNKETT D. (2003b) : Civil Renewal: A New Agenda. London: CSV/Home Office. CRAIG R., KERR D., WADE P. and TAYLOR G. (2005): Taking Post-16 Citizenship Forward: Learning from the Post-16 Citizenship Development Projects. (DfES Research Report 604). London: DfES.

CRICK B. (1998): Education for Citizenship and the Teaching of Democracy in Schools: Final report of the advisory group on citizenship. London: QCA.

DEAKIN CRICK R., COATES M., TAYLOR M. and RITCHIE S. (2004) : A Systematic Review of the Impact of Citizenship Education on the Provision of Schooling. London: EPPI - Centre, University of London.

Department for Education and Skills (2007): Diversity and Citizenship: Curriculum Review. London: DfES.

Further Education Funding Council (2000): Citizenship for 16-19 Year Olds in Education and Training: Report of the Advisory Group to the Secretary of State for Education and Employment. Coventry: FEFC.

IRELAND E., KERR D., LOPES J. and NELSON J. with CLEAVER E. (2006): Active Citizenship and Young People: Opportunities, Experiences and Challenges in and Beyond School, Citizenship Education Longitudinal Study: Fourth Annual Report. (DfES Research Report 732). London: DfES. 
KERR D. (1999a): Re-examining Citizenship Education: The Case of England. Slough: NFER.

KERR D., LINES A., BLENKINSOP S. and SCHAGEN I. (2002): What Citizenship and Education Mean to 14-Year Olds: England's Results from the IEA Citizenship Education Study. London: DfES/NFER.

KERR D., IRELAND E., LOPES J. and CRAIG R. with CLEAVER E. (2004): Making Citizenship Real. Citizenship Education Longitudinal Study Second Annual Report. First Longitudinal Survey. (DfES Research Report 531). London: DfES.

KERR D. and CLEAVER E. (2004): Citizenship Education Longitudinal Study: Literature Review - Citizenship Education One Year On - What Does it Mean?: Emerging Definitions and Approaches in the First Year of National Curriculum Citizenship in England. (DfES Research Report 532). London: DfES.

MAYLOR U. and READ B. with MENDICK H., ROSS A. and ROLLOCK N. (2007): Diversity and Citizenship in the Curriculum: Research Review. (DfES Research Report 819). London: DfES.

OFSTED (2006): Towards Consensus: Citizenship in Secondary Schools. HMI Report 2666. London: OFSTED.

Qualifications and Curriculum Authority (1999): Citizenship: Key Stages 3-4. London: DFEE/QCA.

Qualifications and Curriculum Authority (2003): Citizenship: 2002-2003 Annual Report by QCA's Diversity and Inclusion Team. London: QCA.

TORNEY-PURTA J., LEHMANN R., OSWALD H. and SCHULZ W. (2001) : Citizenship and Education in Twenty-Eight Countries: Civic Knowledge and Participation at Age Fourteen. Amsterdam: International Association for the Evaluation of Educational Achievement (IEA). 